\title{
STOCHASTIC ANTIDERIVATIONAL EQUATIONS ON NON-ARCHIMEDEAN BANACH SPACES
}

\author{
S. V. LUDKOVSKY
}

Received 28 October 2002

\begin{abstract}
Stochastic antiderivational equations on Banach spaces over local non-Archimedean fields are investigated. Theorems about existence and uniqueness of the solutions are proved under definite conditions. In particular, Wiener processes are considered in relation to the non-Archimedean analog of the Gaussian measure.
\end{abstract}

2000 Mathematics Subject Classification: 28C20, 46S10.

1. Introduction. This paper continues the investigations of stochastic processes on non-Archimedean spaces [8]. In the first part, stochastic processes were defined on Banach spaces over non-Archimedean local fields and the analogs of Itô formula were proved. This part is devoted to stochastic antiderivational equations. In the non-Archimedean case, antiderivational equations are used instead of stochastic integral or differential equations in the classical case.

In Section 2, suitable analogs of Gaussian measures are considered. Certainly they do not have any complete analogy with the classical one, some of their properties are similar and some are different. They are used for the definition of the standard (Wiener) stochastic process. Integration by parts formula for the non-Archimedean stochastic processes is studied. Some particular cases of the general Itô formula from [8] are discussed here more concretely. In Section 3, with the help of them, stochastic antiderivational equations are defined and investigated. Analogs of theorems about existence and uniqueness of solutions of stochastic antiderivational equations are proved. Generating operators of solutions of stochastic equations are investigated. All results of this paper are obtained for the first time.

In this paper the notations of [8] are also used.

\section{Gaussian measures and standard Wiener processes on a non-Archimedean Banach space}

2.1. Let $H=c_{0}(\alpha, \mathbf{K})$ be a Banach space over a local field $\mathbf{K}$. Let $U^{P}$ be a cylindrical algebra generated by projections on finite-dimensional over $\mathbf{K}$ subspaces $F$ in $H$ and Borel $\sigma$-algebras $B f(F)$. Denote by $u$ the minimal $\sigma$-algebra $\sigma\left(u^{P}\right)$ generated by $u^{P}$. 
We consider functions, whose Fourier transform has the form

$$
\hat{f}(x)=\hat{f}_{\beta, \gamma, q}(x):=\exp \left(-\beta|x|^{q}\right) \chi_{\gamma}(x),
$$

where the Fourier transform was defined in [12] and [13, Section 7], $\gamma \in \mathbf{K}$, $0<\beta<\infty, 0<q<\infty$.

DEFINITION 2.1. A cylindrical measure $\mu$ on $u^{P}$ is called $q$-Gaussian if each of its one-dimensional projections is $q$-Gaussian, that is,

$$
\mu^{g}(d x)=C_{\beta, \gamma, q} f_{\beta, \gamma, q} v(d x),
$$

where $v$ is the Haar measure on $B f(\mathbf{K})$ with values in $\mathbb{R}, g$ is a continuous K-linear functional on $H=c_{0}(\alpha, \mathbf{K})$ giving projection on one-dimensional subspace in $H, C_{\beta, \gamma, q}>0$ are constants such that $\mu^{g}(\mathbf{K})=1, \beta$ and $\gamma$ may depend on $g, q$ is independent of $g$ where $1 \leq q<\infty$, and $\alpha \subset \omega_{0}$ where $\omega_{0}$ is the first countable ordinal.

If $\mu$ is a measure on $H$, then $\hat{\mu}$ denotes its characteristic functional, that is, $\hat{\mu}(g):=\int_{H} \chi_{g}(x) \mu(d x)$, where $g \in H^{*}, \chi_{g}: H \rightarrow \mathbb{C}$ is the character of $H$ as the additive group (see [8, Section 3.4]).

THEOREM 2.2. A nonnegative $q$-Gaussian measure $\mu$ on $c_{0}\left(\omega_{0}, \mathbf{K}\right)$ is $\sigma$-additive on $B f\left(c_{0}\left(\omega_{0}, \mathbf{K}\right)\right)$ if and only if there exists an injective compact operator $J \in L_{q}\left(c_{0}\left(\omega_{0}, \mathbf{K}\right)\right)$ for a chosen $1 \leq q<\infty$ such that

$$
\mu(d x)=\bigotimes_{j=1}^{\infty} \mu_{j}\left(d x^{j}\right)
$$

where

$$
\begin{gathered}
J=\operatorname{diag}\left(\zeta_{j}: \zeta_{j} \in \mathbf{K}, j \in \omega_{0}\right), \\
\mu_{j}\left(d x^{j}\right)=C_{\beta_{j}, \gamma_{j}, q} f_{\beta_{j}, \gamma_{j}, q} v\left(d x^{j}\right)
\end{gathered}
$$

are measures on $e_{j} \mathbf{K}, x=\left(x^{j}: j \in \omega_{0}\right) \in c_{0}\left(\omega_{0}, \mathbf{K}\right), x^{j} \in \mathbf{K}, \beta_{j}=\left|\zeta_{j}\right|^{-q}$, and $\gamma=\left(\gamma_{j}: j \in \omega_{0}\right) \in c_{0}\left(\omega_{0}, \mathbf{K}\right)$. Moreover, each one-dimensional projection $\mu^{g}$ has the following characteristic functional:

$$
\hat{\mu}^{g}(h)=\exp \left(-\left(\sum_{j} \beta_{j}\left|g_{j}\right|^{q}\right)|h|^{q}\right) \chi_{g(\gamma)}(h),
$$

where $g=\left(g_{j}: j \in \omega_{0}\right) \in c_{0}\left(\omega_{0}, \mathbf{K}\right)^{*}$.

Proof. Let $\theta$ be a characteristic functional of $\mu$. By the non-Archimedean analog of the Minlos-Sazonov theorem (see [6, Section 2.31], [5, 7]), a measure $\mu$ is $\sigma$-additive if and only if, for each $c>0$, there exists a compact operator 
$S_{c}$ such that $|\operatorname{Re}(\mu(y)-\mu(x))|<c$ for each $x, y \in c_{0}\left(\omega_{0}, \mathbf{K}\right)$ with $\left|z^{*} S_{c} z\right|<1$, where $z=x-y$. From Definition 2.1, it follows that each projection $\mu_{j}$ on $\mathbf{K} e_{j}$ has the form given by (2.5). It remains to establish that $\mu$ is $\sigma$-additive if and only if $J \in L_{q}\left(c_{0}\left(\omega_{0}, \mathbf{K}\right)\right)$ and $\gamma \in c_{0}\left(\omega_{0}, \mathbf{K}\right)$.

We have

$$
\begin{aligned}
\mu_{j}(\mathbf{K} \backslash B(\mathbf{K}, 0, r)) & \leq C \int_{x \in \mathbf{K},|x|>r} \exp \left(-\left|\frac{x}{\zeta_{j}}\right|^{q}\right)\left|\zeta_{j}\right|^{-1} v(d x) \\
& \leq C_{1} \int_{y \in \mathbb{R},|y|>r} \exp \left(-|y|^{q}\left|\zeta_{j}\right|^{-q}\right)\left|\zeta_{j}\right|^{-1} d y,
\end{aligned}
$$

where $C>0$ and $C_{1}>0$ are constants independent of $\zeta_{j}$ for $b_{0}>p^{3}$ and each $r>b_{0}, 1 \leq q<\infty$ is fixed (see also the proof of [6, Lemma 2.8], [7], and [2, Theorem II.2.1]). Evidently, $g(\gamma)$ is correctly defined for each $g \in c_{0}\left(\omega_{0}, \mathbf{K}\right)^{*}$ if and only if $\gamma \in \mathcal{c}_{0}\left(\omega_{0}, \mathbf{K}\right)$. In this case the character $\chi_{g(\gamma)}: \mathbf{K} \rightarrow \mathbb{C}$ is defined and $\chi_{g}(\gamma)=\prod_{j=1}^{\infty} \chi_{g_{j} \gamma_{j}}$. Due to [6, Lemma 2.3] and [7], if $J \in L_{q}\left(c_{0}\right)$ and $\gamma \in$ $c_{0}\left(\omega_{0}, \mathbf{K}\right)$, then $\mu$ is $\sigma$-additive.

Let $0 \neq g \in c_{0}^{*}$. Since $\mathbf{K}$ is the local field, there exists $x_{0} \in c_{0}$ such that $\left|g\left(x_{0}\right)\right|=\|g\|$ and $\left\|x_{0}\right\|=1$. Put $g_{j}:=g\left(e_{j}\right)$. Then $\|g\| \leq \sup _{j}\left|g_{j}\right|$ since $g(x)=$ $\sum_{j} x^{j} g_{j}$, where $x=x^{j} e_{j}:=\sum_{j} x^{j} e_{j}$ with $x^{j} \in \mathbf{K}$. Consequently, $\|g\|=\sup _{j}\left|g_{j}\right|$. We denumerate the standard orthonormal basis $\left\{e_{j}: j \in \mathbb{N}\right\}$ such that $\left|g_{1}\right|=$ $\|g\|$. There exists an operator $E$ on $c_{0}$ with matrix elements $E_{i, j}=\delta_{i, j}$ for each $i, j>1, E_{1, j}=g_{j}$ for each $j \in \mathbb{N}$. Then $\left|\operatorname{det} P_{n} E P_{n}\right|=\|g\|$ for each $n \in \mathbb{N}$, where $P_{n}$ are the standard projectors on $\operatorname{sp}_{\mathbf{K}}\left\{e_{1}, \ldots, e_{n}\right\}$ [9]. When $g \in\left\{e_{j}^{*}: j \in \omega_{0}\right\}$, then evidently, $\mu^{g}$ has the form given by (2.5) since $\mu_{i}(\mathbf{K})=1$ for each $i \in \omega_{0}$, where $e_{j}^{*}\left(e_{i}\right)=\delta_{i, j}$ for each $i, j$.

Suppose now that $J \notin L_{q}\left(c_{0}\right)$. For this, we consider $\mu^{g}(\mathbf{K} \backslash B(\mathbf{K}, 0, r)) \geq$ $\sum_{j} \int_{x \in \mathbf{K},|x|>r} C \exp \left(-\left|x / \zeta_{j}\right|^{q}\right)\left|\zeta_{j}\right|^{-1} v(d x)$, where $g=(1,1,1, \ldots) \in c_{0}^{*}=l^{\infty}\left(\omega_{0}\right.$, $\mathbf{K})$. On the other hand, there exists a constant $C_{2}>0$ such that for $b_{0}>p^{3}$ and for each $r>b_{0}$, we have the following inequality:

$$
\begin{array}{r}
\int_{x \in \mathbf{K},|x|>r} C \exp \left(-\left|\frac{x}{\zeta_{j}}\right|^{q}\right)\left|\zeta_{j}\right|^{-1} v(d x) \\
\geq C_{2}\left[\int_{r}^{\infty} \exp \left(-|y|^{q}\left|\zeta_{j}\right|^{-q}\right)\left|\zeta_{j}\right|^{-1} d y\right. \\
\left.\quad+\int_{-\infty}^{-r} \exp \left(-|y|^{q}\left|\zeta_{j}\right|^{-q}\right)\left|\zeta_{j}\right|^{-1} d y\right] .
\end{array}
$$

From the estimates of [2, Lemma II.1.1] and using the substitution $z=y^{1 / 2 q}$ for $y>0$ and $z=(-y)^{1 / 2 q}$ for $y<0$, we get that $\mu^{g}$ is not $\sigma$-additive, consequently, $\mu$ is not $\sigma$-additive since $P_{g}^{-1}(A)$ are cylindrical Borel subsets for each $A \in B f(\mathbf{K})$, where $P_{g} z=g(z)$ is the induced projection on $\mathbf{K}$ for each $z \in c_{0}$. 
For the verification of formula (2.6), it is sufficient at first to consider the measure $\mu$ on the algebra $U^{P}$ of cylindrical subsets in $c_{0}$. Then for each projection $\mu^{g}$, where $g \in \operatorname{sp}_{\mathbf{K}}\left(e_{1}, \ldots, e_{m}\right)^{*}$, we have

$$
\hat{\mu}^{g}(h)=\int_{\mathbf{K}} \cdots \int_{\mathbf{K}} x_{e}(h z) \mu_{1}\left(d x_{1}\right) \cdots \mu_{m}\left(d x_{m}\right),
$$

where $e=(1, \ldots, 1) \in \mathbf{Q}_{p}^{n}, h \in \mathbf{K}, n:=\operatorname{dim}_{\mathrm{Q}_{p}} \mathbf{K}, x^{i} \in \mathbf{K} e_{i}, z=g(x), x=\left(x^{1}, \ldots\right.$, $\left.x^{m}\right)$, consequently, $\hat{\mu}^{g}(h)=\prod_{i=1}^{m} \hat{\mu}_{i}\left(h g_{i}\right)$ since $\chi_{e}(h g(x))=\prod_{i=1}^{m} \chi_{e}\left(h_{i} g_{i} x^{i}\right)$ for each $x \in \operatorname{sp}_{\mathbf{K}}\left(e_{1}, \ldots, e_{m}\right)$. Since $J \in L_{q}$, then $\mu$ is the Radon measure, consequently, the continuation of $\mu$ from $u^{P}$ produces $\mu$ on the Borel $\sigma$-algebra of $c_{0}$, hence $\lim _{m \rightarrow \infty} \hat{\mu}^{Q_{m} g}(h)=\hat{\mu}^{g}(h)$, where $Q_{m}$ is the natural projection on $\operatorname{sp}_{\mathbf{K}}\left(e_{1}, \ldots, e_{m}\right) *$ for each $m \in \mathbb{N}$ such that $Q_{m}(g)=\left(g_{1}, \ldots, g_{m}\right)$. Using expressions of $\hat{\mu}_{i}$, we get formula (2.6). From this, it follows that if $J \in L_{q}$, then $\hat{\mu}(g)$ exists for each $g \in c_{0}^{*}$ if and only if $\gamma \in c_{0}$, since $\hat{\mu}^{g}(h)=\hat{\mu}(g h)$ for each $h \in \mathbf{K}$ and $g \in c_{0}^{*}$.

COROLLARY 2.3. For each $h_{1}, h_{2} \in \mathbf{K}$ and $g \in c_{0}\left(\omega_{0}, \mathbf{K}\right)^{*},\left|\hat{\mu}^{g}\left(h_{1}+h_{2}\right)\right| \leq$ $\max \left(\left|\hat{\mu}^{g}\left(h_{1}\right)\right|,\left|\hat{\mu}^{g}\left(h_{2}\right)\right|\right)$.

REMARK 2.4. Let $Z$ be a compact subset without isolated points in a local field $\mathbf{K}$, for example, $Z=B\left(\mathbf{K}, t_{0}, 1\right)$. Then the Banach space $C^{0}(Z, \mathbf{K})$ has the Amice polynomial orthonormal base $Q_{m}(x)$, where $x \in Z, m \in \mathbb{N}_{0}:=$ $\{0,1,2, \ldots\}$ [1]. Suppose that $\tilde{P}^{n-1}: C^{n-1}(Z, \mathbf{K}) \rightarrow C^{n}(Z, \mathbf{K})$ are antiderivations from [11, Section 80], where $n \in \mathbb{N}$. Each $f \in C^{0}$ has a decomposition $f(x)=$ $\sum_{m} a_{m}(f) Q_{m}(x)$, where $a_{m} \in \mathbf{K}$. These decompositions establish the isometric isomorphism $\theta: C^{0}(Z, \mathbf{K}) \rightarrow c_{0}\left(\omega_{0}, \mathbf{K}\right)$ such that $\|f\|_{C^{0}}=\max _{m}\left|a_{m}(f)\right|=$ $\|\theta(f)\|_{c_{0}}$. Since $Z$ is homeomorphic with $\mathbf{Z}_{p}$, then $\tilde{P}^{1} \tilde{P}^{0}: C^{0}(Z, \mathbf{K}) \rightarrow C^{2}(Z, \mathbf{K})$ is a linear injective compact operator such that $\tilde{P}^{1} \tilde{P}^{0} \in L_{1}$, where $\tilde{P}^{j}$ here corresponds to $\tilde{P}_{j+1}: C^{j} \rightarrow C^{j+1}$ antiderivation operator by Schikhof (see also [11, Sections 54 and 80] and [6, Section I.2.1]). The Banach space $C^{2}(Z, \mathbf{K})$ is dense in $C^{0}(Z, \mathbf{K})$. Using Theorem 2.2 above and [8, Note 2.3] for $q \geq 1$, we get a $q$-Gaussian measure on $C^{0}(Z, \mathbf{K})$, where $\tilde{P}^{1} \tilde{P}^{0} f=\sum_{j} \lambda_{j} P_{j} f$ and $J f=\sum_{j} \zeta_{j} P_{j} f$ for each $f \in C^{0}$, we put $\left|\lambda_{j}\right||\pi|^{q} \leq\left|\zeta_{j}\right|^{q} \leq\left|\lambda_{j}\right|$ for each $j \in \mathbb{N}, P_{j}$ are projectors, $\lambda_{j}, \zeta_{j} \in \mathbf{K}, p^{-1} \leq|\pi|<1, \pi \in \mathbf{K}$, and $|\pi|$ is the generator of the valuation group of $\mathbf{K}$.

If $H=c_{0}\left(\omega_{0}, \mathbf{K}\right)$, then the Banach space $C^{0}(Z, H)$ is isomorphic with the tensor product $C^{0}(Z, \mathbf{K}) \otimes H$ (see [12, Section 4.R]). Therefore, the antiderivation $\tilde{P}^{n}$ on $C^{n}(Z, \mathbf{K})$ induces the antiderivation $\tilde{P}^{n}$ on $C^{n}(Z, H)$. If $J_{i} \in L_{q}\left(Y_{i}\right)$, then $J:=J_{1} \otimes J_{2} \in L_{q}\left(Y_{1} \otimes Y_{2}\right)$ (see also [12, Theorem 4.33]). Put $Y_{1}=C^{0}(Z, \mathbf{K})$ and $Y_{2}=H$, then each $J:=J_{1} \otimes J_{2} \in L_{q}\left(Y_{1} \otimes Y_{2}\right)$ induces the $q$-Gaussian measure $\mu$ on $C^{0}(Z, H)$ such that $\mu=\mu_{1} \otimes \mu_{2}$, where $\mu_{i}$ are $q$-Gaussian measures on $Y_{i}$ induced by $J_{i}$ as above. In particular, for $q=1$ we can also take $J_{1}=$ $\tilde{P}^{1} \tilde{P}^{0}$. The 1-Gaussian measure on $C^{0}(Z, H)$ induced by $J=J_{1} \otimes J_{2} \in L_{1}$ with $J_{1}=\tilde{P}^{1} \tilde{P}^{0}$ is called standard. Analogously considering the following Banach 
subspace $C_{0}^{0}(Z, H):=\left\{f \in C^{0}(Z, H): f\left(t_{0}\right)=0\right\}$ and operators $J:=J_{1} \otimes J_{2} \in$ $L_{1}\left(C_{0}^{0}(Z, \mathbf{K}) \otimes H\right)$, we get the 1 -Gaussian measures $\mu$ on it also, where $t_{0} \in Z$ is a marked point. Certainly, we can take other operators $J_{1} \in L_{q}\left(Y_{1}\right)$ not related with the antiderivation as above.

\section{Non-Archimedean stochastic antiderivational equations}

3.1. We define a (non-Archimedean) Wiener process $w(t, \omega)$ with values in $H$ as a stochastic process (see [8, Definition 4.1]) such that

(ii) ${ }^{\prime}$ the random variable $w(t, \omega)-w(u, \omega)$ has a distribution $\mu^{F_{t, u}}$, where $\mu$ is a probability Gaussian measure on $C^{0}(T, H)$ described in Definition 2.1.

3.2. If $\mu$ is the standard Gaussian measure on $C_{0}^{0}(T, H)$, then the Wiener process is called standard (see also [6, Theorem 3.23, Lemmas 2.3, 2.5, 2.8, and Section 3.30], [7]).

REMARK 3.1. In [8] the non-Archimedean analogs of the Itô formula were proved. In the particular case $H=\mathbf{K}$ we have $a \in L^{s}\left(\Omega, \mathscr{F}, \lambda ; C^{0}(T, \mathbf{K})\right), E \in$ $L^{r}\left(\Omega, \mathscr{F}, \lambda ; C^{0}(T, \mathbf{K})\right), f \in C^{n}(T \times \mathbf{K}, Y)$, and $w \in L^{q}\left(\Omega, \mathscr{F}, \lambda ; C_{0}^{0}(T, \mathbf{K})\right)$ are functions (see [8, Sections 3.1, 4.3 and Definition 4.1]), so that

$$
\begin{gathered}
\left.\hat{P}_{u^{b+m-l}, w(u, \omega)^{l}}\left[\left(\frac{\partial^{m+b} f}{\partial u^{b} \partial x^{m}}\right)(u, \xi(u, \omega)) \circ\left(I^{\otimes b} \otimes a^{\otimes(m-l)} \otimes E^{\otimes l}\right)\right]\right|_{u=t} \\
=\sum_{j}\left(\frac{\partial^{m+b} f}{\partial u^{b} \partial x^{m}}\right)\left(t_{j}, \xi\left(t_{j}, \omega\right)\right)\left[t_{j+1}-t_{j}\right]^{b+m-l} a\left(t_{j}, \omega\right)^{k-l} \\
\times\left[E\left(t_{j}, \omega\right)\left(w\left(t_{j+1}, \omega\right)-w\left(t_{j}, \omega\right)\right)\right]^{l}
\end{gathered}
$$

for each $m+b \leq n$, where $t_{j}=\sigma_{j}(t)$ and $a(t, \omega), E(t, \omega), w(t, \omega) \in \mathbf{K}$, that is, $a, E, w$ commute. In particular, $\tilde{P}_{u, 0}^{m} f(u)=\sum_{k=1}^{m}(k !)^{-1} \hat{P}_{u^{k}} f^{(k)}(u)$, that is, $\left.\tilde{P}_{u, 0}^{m} f(u)\right|_{u=t}=\tilde{P}_{m+1} f^{\prime}(t)$, where $\tilde{P}_{m+1}: C^{m}(T, \mathbf{K}) \rightarrow C^{m+1}(T, \mathbf{K})$ is the Schikhof linear continuous antiderivation operator (cf. [11, Section 80]).

In the non-Archimedean case, the formula

$$
M\left[\left(\int_{S}^{T} \phi(t, \omega) d B_{t}(\omega)\right)^{2}\right]=M\left[\int_{S}^{T} \phi(t, \omega)^{2} d t\right]
$$

(see [10, Lemma 3.5]) is not valid, but it has another analog. Let $X$ be a locally compact Hausdorff space and let $B C_{C}(X, H)$ denote a subspace of $C^{0}(X, H)$ consisting of bounded continuous functions $f$ such that for each $\epsilon>0$ there exists a compact subset $V \subset X$ for which $\|f(u)\|_{H}<\epsilon$ for each $u \in X \backslash V$. In particular, for $X \subset \mathbf{K}, e^{*} \in H^{*}$, and a fixed $t \in X$ in accordance with [12, Theorem 7.22], there exists a K-valued tight measure $\mu_{t, \omega, e^{*}, b, k}$ on the $\sigma$-algebra 
$\operatorname{Bco}(X)$ of clopen subsets in $X$ such that

$$
\begin{aligned}
& \left.e^{*} \hat{P}_{u^{b}, w^{k}} \psi(u, x, \omega) \circ\left(I^{\otimes b} \otimes E^{\otimes k}\right)\right|_{u=t} \\
& \quad=\int_{X} \psi(u, E(u, \omega) w(u, \omega), \omega) \mu_{t, \omega, e^{*}, b, k}(d u)
\end{aligned}
$$

for each $\psi \in L^{r}\left(\Omega, \mathscr{F}_{F}, \lambda ; B C_{\mathcal{C}}\left(X, L_{k}\left(H^{\otimes k}, H\right)\right)\right)$ and $E \in L^{q}\left(\Omega, \mathscr{F}, \lambda ; B C_{c}(X, L(H))\right)$, where $H^{*}$ is a topologically conjugate space, $1 \leq r, q \leq \infty$, and $1 / r+1 / q \geq 1$.

If $\chi_{y}: \mathbf{K} \rightarrow S^{1}:=\{z \in \mathbb{C}:|z|=1\}$ is a continuous character of $\mathbf{K}$ as the additive group, then

$$
\begin{aligned}
M \chi_{\gamma} & \left(\left(\left.e^{*} \hat{P}_{u^{b}, w^{k}} \psi(u, x, \omega) \circ\left(I^{\otimes b} \otimes E^{\otimes k}\right)\right|_{u=t}\right)^{l}\right) \\
= & \prod_{j} M \chi_{\gamma}\left(\left(e^{*} \psi\left(t_{j}, x, \omega\right)\left[t_{j+1}-t_{j}\right]^{b}\right)\right. \\
& \left.\circ\left(1^{\otimes b} \otimes\left(E\left(t_{j}, \omega\right)\left[w\left(t_{j+1}, \omega\right)-w\left(t_{j}, \omega\right)\right]\right)^{\otimes k}\right)^{l}\right)
\end{aligned}
$$

due to [8, Definition 4.1(i)]. For $\psi$ independent from $x, l=1, k=2, b=0, E=1$, and $H=\mathbf{K}$ (so that $e^{*}=1$ ), (3.4) takes a simpler form, which can be considered as another analog of the classical formula. For the evaluation of appearing integrals, tables from [13, Section 1.5.5] can be used. Another important result is the following theorem.

THEOREM 3.2. Let $\psi \in L^{2}\left(\Omega, \mathscr{F}, \lambda ; C^{0}(T, L(H))\right), w \in L^{2}\left(\Omega, \mathscr{F}, \lambda ; C_{0}^{0}(T, H)\right)$ be the stochastic processes on the Banach space $H$ over $\mathbf{K}$. Then there exists a function $\phi \in C^{0}(T, H)$ such that $M \chi_{\gamma}\left(\left.g \hat{P}_{w(u, \omega)} \psi(u, \omega) \circ I\right|_{u=t}\right)=\hat{\mu}\left(\left.\gamma g \hat{P}_{u} \phi(u)\right|_{u=t}\right)$ for each $\gamma \in \mathbf{K}$ and each $t \in T$ and for each $g \in H^{*}$.

Proof. Let $t \in T$ and $t_{j}=\sigma_{j}(t)$, where $\sigma_{j}$ is the approximation of the identity in $T$ and $F_{a, b}(w):=w(a, \omega)-w(b, \omega)$ for $a, b \in T$ (see [8, Section 2.1]). In view of [8, Definition 4.2.(i), (ii)] and the Hahn-Banach theorem [12], there exists a projection operator $\operatorname{Pr}_{g}$ such that $\hat{\mu}^{\left(F_{a, b} g E\right)}(h)=\hat{\mu}^{\left(F_{a, b} \operatorname{Pr}_{g}\right)}\left(\operatorname{Pr}_{g} E h\right)$ since $F_{a, b} g h E w=g h E(w(a, \omega)-w(b, \omega))=h g E F_{a, b} w$ for each $a, b \in T$ and for each $h \in \mathbf{K}$, where $\hat{\mu}$ is the characteristic functional of the measure $\mu$ corresponding to $w$, that is, $\hat{\mu}(g):=\int_{C_{0}^{0}(T, H)} \chi_{g}(y) \mu(d y)$, where $g \in C_{0}^{0}(T, H)^{*}$, $\chi_{g}: C_{0}^{0}(T, H) \rightarrow \mathbb{C}$ is the character of $C_{0}^{0}(T, H)$ as the additive group, $E \in$ $L(H), y \in C_{0}^{0}(T, H)$, and $\mu$ is the Borel measure on $C_{0}^{0}(T, H)$ (see also [8, Section 3.4]). The random variable $E(w(a, \omega)-w(b, \omega))$ has the distribution $\mu^{F_{a, b} E}$ for each $a \neq b \in T$ and $E \in L(H)$. On the other hand, the projection operator $\operatorname{Pr}_{e}$ commutes with the antiderivation operator $\hat{P}_{u}$ on $C^{0}(T, H)$, where $\left(\operatorname{Pr}_{e} f\right)(t):=\operatorname{Pr}_{e} f(t)$ is defined pointwise for each $f \in C^{0}(T, H)$. In $L^{2}\left(\Omega, \mathscr{F}, \lambda ; C^{0}(T, H)\right)$ the family of step functions $f(t, \omega)=\sum_{j=1}^{n} \mathrm{Ch}_{U_{j}}(\omega) f_{j}(t)$ is dense, where $f_{j} \in C^{0}(T, H), \mathrm{Ch}_{U}$ is the characteristic function of $U \in \mathscr{F}_{\text {, }}$ 
and $n \in \mathbb{N}$ since $\lambda(\Omega)=1$ and $\lambda$ is nonnegative. For each $t \in T$, there exists $\lim _{j \rightarrow \infty} \psi\left(t_{j}, \omega\right) \cdot\left(w\left(t_{j+1}, \omega\right)-w\left(t_{j}, \omega\right)\right)$ in $L^{2}(\Omega, \mathscr{F}, \lambda ; H)$ (see [8, Theorem 2.12]).

If $A \in L(H)$, then

(i) $\chi_{\gamma}\left(\left(g_{1}+g_{2}\right) A z\right)=\chi_{\gamma}\left(g_{1} A z\right) \chi_{\gamma}\left(g_{2} A z\right)$ for each $g_{1}, g_{2} \in H^{*}$ and $z \in H$,

(ii) $\chi_{\gamma}\left(g A\left(z_{1}+z_{2}\right)\right)=\chi_{\gamma}\left(g A z_{1}\right) \chi_{\gamma}\left(g A z_{2}\right)$ for each $g \in H^{*}$ and $z_{1}, z_{2} \in H$,

(iii) $\chi_{\gamma}(a g A z)=\left[\chi_{\gamma}(g A z)\right]^{\zeta(a)}$ for each $\{(e, \gamma g A z)\}_{p} \neq 0$ and $a \in \mathbf{K}$, where $\zeta(a):=\{(e, \gamma a g A z)\}_{p} /\{(e, \gamma g A z)\}_{p}$. On the other hand, $A$ is completely defined by the family $\left\{e_{i}^{*} A e_{j}: i, j \in \alpha\right\}$, where $H=c_{0}(\alpha, \mathbf{K}), e_{i}^{*}\left(e_{j}\right)=$ $\delta_{i, j}, e_{i}^{*} \in H^{*}$, and $\left\{e_{j}: j \in \alpha\right\}$ is the standard orthonormal base of $H$. Hence, the family $\left\{\chi_{\gamma}\left(a e_{i}^{*} A e_{j}\right): i, j \in \alpha ; a \in \mathbf{K}\right\}$ completely characterizes $A \in L(H)$ due to (i), (ii), and (iii) when $\gamma \neq 0$.

For each $y \in H$ and each $\gamma \in \mathbf{K}$, the function $M \chi_{\gamma}(g \psi(t, \omega) y)$ is continuous by $t \in T$, consequently, there exists a continuous function $\phi: T \rightarrow H$ such that $M \chi_{\gamma}(g \psi(t, \omega) y)=\chi_{\gamma}(g \phi(t) y)$ for each $y \in H$ and $t \in T$ since characters $\chi_{\gamma}$ are continuous from $\mathbf{K}$ to $\mathbb{C}$ and $\chi_{\gamma}(h)=\chi_{1}(\gamma h)$ for each $0 \neq \gamma \in \mathbf{K}$ and $h \in \mathbf{K}$ and the $\mathbb{C}$-linear span of the family $\left\{\chi_{\gamma}: \gamma \in \mathbf{K}\right\}$ of characters is dense in $C^{0}(\mathbf{K}, \mathbb{C})$ by the Stone-Weierstrass theorem [3, 4]. On the other hand,

$$
\lim _{j \rightarrow \infty} \chi_{\gamma}\left(\sum_{i=0}^{j} a_{j}\right)=\prod_{i=1}^{\infty} \chi_{\gamma}\left(a_{i}\right)
$$

when $\lim _{j} a_{j}=0$ for a sequence $a_{j}$ in $\mathbf{K}$. Therefore,

$$
\begin{aligned}
M \chi_{\gamma}\left(g \sum_{j=0}^{\infty} \psi\left(t_{j}, \omega\right) \cdot\left[w\left(t_{j+1}, \omega\right)-w\left(t_{j}, \omega\right)\right]\right) \\
=\prod_{j=0}^{\infty} \hat{\mu}\left(\gamma g \phi\left(t_{j}\right)\left(t_{j+1}-t_{j}\right)\right) \\
=\hat{\mu}\left(\left.\gamma g \hat{P}_{u} \phi(u)\right|_{u=t}\right) \quad \text { for each } t \in T \text { and each } g \in H^{*} .
\end{aligned}
$$

From the equality $\chi_{a+b}(c)=\chi_{a}(c) \chi_{b}(c)$ for each $a, b, c \in \mathbf{K}$, the statement of this theorem follows for each $\gamma \in \mathbf{K}$.

THEOREM 3.3. Let $a \in L^{q}\left(\Omega, \mathscr{F}, \lambda ; C^{0}\left(B_{R}, L^{q}\left(\Omega, \mathscr{F}, \lambda ; C^{0}\left(B_{R}, H\right)\right)\right)\right)$ and $E \in$ $L^{r}\left(\Omega, \mathscr{F}, \lambda ; C^{0}\left(B_{R}, L\left(L^{q}\left(\Omega, \mathscr{F}, \lambda ; C^{0}\left(B_{R}, H\right)\right)\right)\right)\right), a=a(t, \omega, \xi), E=E(t, \omega, \xi), t \in$ $B_{R}, \omega \in \Omega, \xi \in L^{q}\left(\Omega, \mathscr{F}, \lambda ; C^{0}\left(B_{R}, H\right)\right)$ and $\xi_{0} \in L^{q}(\Omega, \mathscr{F}, \lambda ; H)$, and $w \in L^{s}(\Omega, \mathscr{F}, \lambda$; $\left.C_{0}^{0}\left(B_{R}, H\right)\right)$ with $1 / r+1 / s=1 / q, 1 \leq r, s, q \leq \infty$, where $a$ and $E$ satisfy the local Lipschitz condition

(LLC) for each $0<r<\infty$ there exists $K_{r}>0$ such that

$$
\max (\|a(t, \omega, x)-a(t, \omega, y)\|,\|E(t, \omega, x)-E(t, \omega, y)\|) \leq K_{r}\|x-y\|
$$


for each $x, y \in B\left(C^{0}\left(B_{R}, H\right), 0, r\right), t \in B_{R}$, and $\omega \in \Omega$. Then the stochastic process of the type

$$
\xi(t, \omega)=\xi_{0}(\omega)+\left.\left(\hat{P}_{u} a\right)(u, \omega, \xi)\right|_{u=t}+\left.\left(\hat{P}_{w(u, \omega)} E\right)(u, \omega, \xi)\right|_{u=t}
$$

has a unique solution.

THEOREM 3.4. Let $a \in L^{\infty}\left(\Omega, \mathscr{F}, \lambda ; C^{0}\left(B_{R}, L^{q}\left(\Omega, \mathscr{F}, \lambda ; C^{0}\left(B_{R}, H\right)\right)\right)\right)$ and $E \in$ $L^{\infty}\left(\Omega, \mathscr{F}, \lambda ; C^{0}\left(B_{R}, L\left(L^{q}\left(\Omega, \mathscr{F}, \lambda ; C^{0}\left(B_{R}, H\right)\right)\right)\right)\right), a=a(t, \omega, \xi), E=E(t, \omega, \xi), t \in$ $B_{R}, \omega \in \Omega, \xi \in L^{q}\left(\Omega, \mathscr{F}, \lambda ; C^{0}\left(B_{R}, H\right)\right)$ and $\xi_{0} \in L^{q}(\Omega, \mathscr{F}, \lambda ; H), w \in L^{\infty}(\Omega$, $\left.\mathscr{F}_{F}, \lambda ; C_{0}^{0}\left(B_{R}, H\right)\right), 1 \leq q \leq \infty$, where $a$ and $E$ satisfy the local Lipschitz condition (LLC). Suppose there is a stochastic process of the type

(i) $\xi(t, \omega)=\xi_{0}(\omega)+\sum_{m+b=1}^{\infty} \sum_{l=0}^{m}\left(\hat{P}_{u^{b+m-l}, w(u, \omega) l}\left[a_{m-l+b, l}(u, \xi(u, \omega)) \circ\right.\right.$ $\left.\left.\left(I^{\otimes b} \otimes a^{\otimes(m-l)} \otimes E^{\otimes l}\right)\right]\right)\left.\right|_{u=t}$ such that $a_{m-l, l} \in C^{0}\left(B_{R_{1}} \times B\left(L^{q}(\Omega, \mathscr{F}, \lambda ;\right.\right.$ $\left.\left.\left.C^{0}\left(B_{R}, H\right)\right), 0, R_{2}\right), L_{m}\left(H^{\otimes m} ; H\right)\right)$ is continuous and bounded on its domain for each $n, l, 0<R_{2}<\infty$,

(ii) $\lim _{n \rightarrow \infty} \sup _{0 \leq l \leq n}\left\|a_{n-l, l}\right\|_{C^{0}\left(B_{R_{1}} \times B\left(L^{q}\left(\Omega, \mathscr{F}, \lambda ; C^{0}\left(B_{R}, H\right)\right), 0, R_{2}\right), L_{n}\left(H^{\otimes n}, H\right)\right)}=0$ for each $0<R_{1} \leq R$ when $0<R<\infty$, for each $0<R_{1}<R$ when $R=\infty$, and for each $0<R_{2}<\infty$.

Then (i) has a unique solution in $B_{R}$.

Proof of Theorem 3.4. We have $\max \left(\|a(x)-a(y)\|^{g},\|E(x)-E(y)\|^{g}\right) \leq$ $K\|x-y\|^{g}$, hence $\max \left(\|a(x)\|^{g},\|E(x)\|^{g}\right) \leq K_{1}\left(\|x\|^{g}+1\right)$ for each $x, y \in H$ and for each $1 \leq g<\infty, t \in B_{R}$, and each $\omega \in \Omega$, where $K$ and $K_{1}$ are positive constants, $a(x)$ and $E(x)$ are short notations of $a(t, \omega, x)$ and $E(t, \omega, x)$ for $x=\xi(t, \omega)$, respectively. Let $X_{0}(t)=x, \ldots$,

$$
\begin{array}{r}
X_{n}(t)=x+\sum_{m+b=1}^{\infty} \sum_{l=0}^{m}\left(\hat{P}_{u^{b+m-l}, w(u, \omega)^{l}}[\right. \\
a_{m-l+b, l}\left(u, X_{n-1}(u, \omega)\right) \\
\left.\left.\circ\left(I^{\otimes b} \otimes a^{\otimes(m-l)} \otimes E^{\otimes l}\right)\right]\right)\left.\right|_{u=t},
\end{array}
$$

consequently,

$$
\begin{aligned}
& X_{n+1}-X_{n}(t)= \\
& \sum_{m+b=1}^{\infty} \sum_{l=0}^{m}\left(\hat{P}_{u^{b+m-l}, w(u, \omega)^{l}}\left[a_{m-l+b, l}\left(u, X_{n}(u)\right)-a_{m-l+b, l}\left(u, X_{n-1}(u)\right)\right]\right. \\
& \left.\circ\left(I^{\otimes b} \otimes a^{\otimes(m-l)} \otimes E^{\otimes l}\right)\right)\left.\right|_{u=t},
\end{aligned}
$$

where in general

$$
\begin{aligned}
\left.\hat{P}_{a(u, \xi)} 1\right|_{u=t} & =a(t, \xi(t, \omega))-a\left(t_{0}, \xi\left(t_{0}, \omega\right)\right) \neq \hat{P}_{u} a(u, \xi) \\
& =\sum_{j} a\left(t_{j}, \xi\left(t_{j}, \omega\right)\right)\left[t_{j+1}-t_{j}\right],
\end{aligned}
$$


$t_{j}=\sigma_{j}(t)$ for each $j=0,1,2, \ldots$ Let $M(\eta)$ be a mean value of a real-valued distribution $\eta(\omega)$ by $\omega \in \Omega$. Then

$$
\begin{aligned}
M \|\left.\hat{P}_{u^{b+m-l}, w(u, w) l}\left[a_{m-l+b, l}\left(u, X_{n}(u)\right)-a_{m-l+b, l}\left(u, X_{n-1}(u)\right)\right]\right|_{\left(B_{R_{1}} \times B\left(L^{q}, 0, R_{2}\right)\right)} & \left.\quad \quad\left(I^{\otimes b} \otimes a^{\otimes(m-l)} \otimes E^{\otimes l}\right)\right|_{u=t} \|^{g} \\
\leq & K\left(M\left\|\hat{P}_{u^{b+m-l}, w(u, w)^{l}}\right\|^{g}\right)\left\|\left.a_{m-l+b, l}\right|_{\left(B_{R_{1}} \times B\left(L^{q}, 0, R_{2}\right)\right)}\right\|^{g} \\
\quad & \quad\left(M \sup _{u}\left\|X_{n}(u)-X_{n-1}(u)\right\|^{g}\right)\left(M \sup _{u}\|a\|^{m-l}\right)\left(M \sup _{u}\|E\|^{l}\right),
\end{aligned}
$$

where $X_{n} \in C_{0}^{0}\left(B_{R}, H\right)$ for each $n, 1 \leq g<\infty$. On the other hand,

$$
\begin{aligned}
& X_{1}(t)=x(t) \\
& \quad+\left.\sum_{m+b=1}^{\infty} \sum_{l=0}^{m}\left(\hat{P}_{u^{b+m-l}, w(u, \omega)^{l}}\left[a_{m-l+b, l}(u, x(u)) \circ\left(I^{\otimes b} \otimes a^{\otimes(m-l)} \otimes E^{\otimes l}\right)\right]\right)\right|_{u=t},
\end{aligned}
$$

consequently,

$$
\begin{aligned}
& \left\|X_{1}(t)-X_{0}(t)\right\|^{g} \\
& \quad \leq\left.\sup _{m, l, b}\left(\left\|\hat{P}_{u^{b+m-l}, w(u, w)^{l}}\left[a_{m-l+b, l}(u, x(u)) \circ\left(I^{\otimes b} \otimes a^{\otimes(m-l)} \otimes E^{\otimes l}\right)\right]\right\|^{g}\right)\right|_{u=t} .
\end{aligned}
$$

Due to condition (ii) of Theorem 3.4 for each $\epsilon>0$ and $0<R_{2}<\infty$, there exists $B_{\epsilon} \subset B_{R}$ such that

$$
\begin{aligned}
K \sup _{m, l, b}\left(\| \hat{P}_{u^{b+m-l},\left.w(u, \omega)^{l}\right|_{B_{\epsilon}}[}\right. & \left.a_{m-l+b, l}(u, *)\right|_{\left(B_{\epsilon} \times B\left(L^{q}, 0, R_{2}\right)\right)} \\
& \left.\left.\circ\left(I^{\otimes b} \otimes a^{\otimes(m-l)} \otimes E^{\otimes l}\right)\right] \|^{g}\right)=: c<1 .
\end{aligned}
$$

Therefore, there exists a unique solution on each $B_{\epsilon}$ since $\sup _{u}\left\|X_{1}(u)-X_{0}(u)\right\|$ $<\infty$ and $\lim _{l \rightarrow \infty} c^{l} C=0$ for each $C>0$, hence there exists $\lim _{n \rightarrow \infty} X_{n}(t)=X(t)=$ $\left.\xi(t, \omega)\right|_{B_{\epsilon}}$, where $C:=M \sup _{u \in B_{\epsilon}}\left\|X_{1}(u)-X_{0}(u)\right\|^{g} \leq(c+1) K<\infty$, here $B_{\epsilon}$ is an arbitrary ball of radius $\epsilon$ in $B_{R}, t \in B_{\epsilon}$.

If $X^{1}$ and $X^{2}$ are two solutions, then $X^{1}-X^{2}=: \psi=\sum_{j=1}^{n} C_{j} C h_{B\left(\mathbf{K}, x_{j}, r_{j}\right)}$, where $n \in \mathbb{N}, C_{j} \in \mathbf{K}, T=B_{R}$, since $B_{R}$ has a disjoint covering by balls $B\left(\mathbf{K}, x_{j}\right.$, $r_{j}$ ), on each such ball there exists a unique solution with a given initial condition on it (i.e., in a chosen point $x_{j}$ such that $C_{j}$ and $B\left(\mathbf{K}, x_{j}, r_{j}\right)$ are independent of $\omega)$. If $S$ is a polyhomogeneous function, then there exists $n=\operatorname{deg}(S)<\infty$ such that differentials $D^{m} S=0$ for each $m>n$, but its antiderivative $\hat{P}$ has $D^{n+1} \hat{P} S \neq 0$. If $\left\|S_{1}\right\|>\left\|S_{2}\right\|$, then $\left\|\hat{P} S_{1}\right\|>\left\|\hat{P} S_{2}\right\|$, which we can apply to a 
convergent series considering terms $\left\|D^{m} \hat{P} S\right\|\left(\bmod p^{k}\right)$ for each $k \in \mathbb{N}$. Therefore,

$$
\begin{gathered}
\psi=\sum_{m+b=1}^{\infty} \sum_{l=0}^{m}\left(\hat{P}_{u^{b+m-l}, w(u, w) l}\left[a_{m-l+b, l}\left(u, X^{2}\right)-a_{m-l+b, l}\left(u, X^{1}\right)\right]\right. \\
\left.\circ\left(I^{\otimes b} \otimes a^{\otimes(m-l)} \otimes E^{\otimes l}\right)\right)\left.\right|_{u=t},
\end{gathered}
$$

where the function $\psi$ is locally constant by $t$ and independent of $\omega$. The term

$$
\left(\Phi^{1} w\right)\left(t_{i} ; 1 ; t_{i+1}-t_{i}\right)=\frac{\left[w\left(t_{i+1}\right)-w\left(t_{i}\right)\right]}{\left(t_{i+1}-t_{i}\right)}
$$

has the infinite-dimensional over $\mathbf{K}$ range in $C^{0}\left(B_{R}^{2} \backslash \Delta, H\right)$ for $\lambda$-a.e. $\omega \in \Omega$, where $\Delta:=\left\{(u, u): u \in B_{R}\right\}$. In view of [8, Lemma 2.2], $\psi=0$ since it is evident for $a(u, X), E(u, X)$, and $a_{k-l, l}(u, X)$ depending on $X$ locally polynomially or polyhomogeneously for each $u$, but such locally polynomial or polyhomogeneous functions by $X$ are dense in

$$
\begin{gathered}
L^{q}\left(\Omega, \mathscr{F}, \lambda ; C^{0}\left(B_{R}, L^{q}\left(\Omega, \mathscr{F}, \lambda ; C^{0}\left(B_{R}, H\right)\right)\right)\right), \\
L^{q}\left(\Omega, \mathscr{F}, \lambda ; C^{0}\left(B_{R}, L\left(L^{q}\left(\Omega, \mathscr{F}, \lambda ; C^{0}\left(B_{R}, H\right)\right)\right)\right),\right. \\
C^{0}\left(B_{R_{1}} \times B\left(L^{q}\left(\Omega, \mathscr{F}, \lambda ; C^{0}\left(B_{R}, H\right)\right), 0, R_{2}\right), L_{k}\left(H^{\otimes k} ; H\right)\right),
\end{gathered}
$$

respectively.

The proof of Theorem 3.3 is a particular case of the latter proof.

Proposition 3.5. Let $\xi$ be the Wiener process given by (3.8) with the 1Gaussian measure associated with the operator $\tilde{P}^{1} \tilde{P}^{0}$ as in Remark 2.4 and let also

$$
\begin{aligned}
\max & (\|a(t, \omega, x)-a(v, \omega, x)\|,\|E(t, \omega, x)-E(v, \omega, x)\|) \\
& \leq|t-v|\left(C_{1}+C_{2}\|x\|^{b}\right)
\end{aligned}
$$

for each $t$ and $v \in B\left(\mathbf{K}, t_{0}, R\right) \lambda$-a.e. by $\omega \in \Omega$, where $b, C_{1}$, and $C_{2}$ are nonnegative constants. Then $\xi$ has a $C^{2}$-modification with probability 1 and

$$
q(t) \leq \max \left\{\left\|\xi_{0}\right\|^{s},\left|t-t_{0}\right|\left(C_{1}+C_{2} q(t)\right)\right\}
$$

for each $t \in B\left(\mathbf{K}, t_{0}, R\right)$, where $q(t):=\sup _{\left|u-t_{0}\right| \leq\left|t-t_{0}\right|} M\|\xi(t, \omega)\|^{s}$ and $\mathbb{N} \ni s \geq$ $b \geq 0$. 
Proof. For the function $f(t, x)=x^{s}$ in accordance with [8, Theorem 4.5], we have

$$
\begin{aligned}
& f(t, \xi(t, \omega)) \\
& =f\left(t_{0}, \xi_{0}\right)+\sum_{k=1}^{s} \sum_{l=0}^{k}\left(\begin{array}{l}
k \\
l
\end{array}\right)\left(\hat{P}_{u^{k-l}, w(u, \omega)^{l}}\right. \\
& \left.\times\left[\left(\begin{array}{l}
s \\
k
\end{array}\right) \xi(t, \omega)^{s-k}(u, \xi(u, \omega)) \circ\left(a^{\otimes(k-l)} \otimes E^{\otimes l}\right)\right]\right)\left.\right|_{u=t},
\end{aligned}
$$

hence

$$
M\|\xi(t, \omega)\|^{s} \leq \max \left(\left\|\xi_{0}\right\|^{s},\left|t-t_{0}\right| d\left(\hat{P}_{*}^{s}\right)\left(C_{1}+C_{2} \sup _{\left|u-t_{0}\right| \leq\left|t-t_{0}\right|} M\|\xi(u, \omega)\|^{s}\right)\right),
$$

since $\left|t_{j}-t_{0}\right| \leq\left|t-t_{0}\right|$ for each $j \in \mathbb{N}$ and

$$
\begin{aligned}
& M\|\xi(t, \omega)-\xi(v, \omega)\|^{s} \\
& \quad \leq|t-v|\left(1+C_{1}+C_{2} d\left(\hat{P}_{*}^{s}\right) \sup _{\left|u-t_{0}\right| \leq \max \left(\left|t-t_{0}\right|,\left|v-t_{0}\right|\right)} M\|\xi(u, \omega)\|^{s}\right),
\end{aligned}
$$

since $\left|t_{j}-v_{j}\right| \leq|t-v|+\rho^{j}$ for each $j \in \mathbb{N}$, where $0<\rho<1$,

$d\left(\hat{P}_{*}^{s}\right):=\frac{\sup _{a \neq 0, E \neq 0, f \neq 0} \max _{s \geq k \geq l \geq 0}\left\|(k !)^{-1}\left(\begin{array}{l}k \\ l\end{array}\right) \hat{P}_{u^{k-l}, w^{l}}\left(\partial^{k} f / \partial^{k} x\right) \circ\left(a^{\otimes(k-l)} \otimes E^{\otimes l}\right)\right\|}{\|a\|_{C^{0}\left(B_{R}, H\right)}^{k-l}\|E\|_{C^{0}\left(B_{R}, L(H)\right)}^{l}\|f\|_{C^{s}\left(B_{R}, H\right)}}$,

hence $d\left(\hat{P}_{*}^{s}\right) \leq 1$, since $f \in C^{s}$ as a function by $x$ and $\left(\bar{\Phi}^{s} g\right)\left(x ; h_{1}, \ldots, h_{s} ; 0, \ldots\right.$, $0)=D_{x}^{s} g(x) \cdot\left(h_{1}, \ldots, h_{s}\right) / s$ ! for each $g \in C^{s}$ and due to the definition of $\|g\|_{C^{s}}$. Considering in particular polyhomogeneous $g$ on which $d\left(\hat{P}_{*}^{s}\right)$ takes its maximum value, we get $d\left(\hat{P}_{*}^{s}\right)=1$. Since $P\left(C^{2}\right)=1$ for the Markov measure $P$ induced by the transition measures $P(v, x, t, S):=\mu^{F_{t, v}}(S \mid \xi(v)=x)$ for $t \neq v$ of the non-Archimedean Wiener process (see Theorem 2.2), then $\xi$ has a $C^{2}$ modification with probability 1 .

NoTE. If we consider a general stochastic process as in [8, Theorem 4.2], then from the proof of Proposition 3.5 it follows that $\xi$ has a modification in the space $J\left(C_{0}^{0}(T, H)\right)$ with the probability 1 , where $J$ is a nondegenerate correlation operator of the product measure $\mu$ on $C_{0}^{0}(T, H)$.

Proposition 3.6. Let $\xi$ be a stochastic process given by (3.8) and let

$$
\begin{aligned}
& \max \left(\left\|a\left(t, \omega, x_{1}\right)-a\left(v, \omega, x_{2}\right)\right\|,\left\|E\left(t, \omega, x_{1}\right)-E\left(v, \omega, x_{2}\right)\right\|\right) \\
& \quad \leq|t-v|\left(C_{1}+C_{2}\left\|x_{1}-x_{2}\right\|^{b}\right)
\end{aligned}
$$


for each $t$ and $v \in B\left(\mathbf{K}, t_{0}, R\right) \lambda$-a.e. by $\omega \in \Omega$, where $b, C_{1}$, and $C_{2}$ are nonnegative constants. Then two solutions $\xi_{1}$ and $\xi_{2}$ with initial conditions $\xi_{1,0}$ and $\xi_{2,0}$ satisfy the following inequality:

$$
y(t) \leq \max \left\{\left\|\xi_{1,0}-\xi_{2,0}\right\|^{s},\left|t-t_{0}\right|\left(C_{1}+C_{2} y(t)\right)\right\}
$$

for each $t \in B\left(\mathbf{K}, t_{0}, R\right)$, where $y(t):=\sup _{\left|u-t_{0}\right| \leq\left|t-t_{0}\right|} M\left\|\xi_{1}(t, \omega)-\xi_{2}(t, \omega)\right\|^{s}$ and $\mathbb{N} \ni s \geq b \geq 0$.

Proof. From Proposition 3.5, it follows that

$$
\begin{aligned}
& M\left\|\xi_{1}(t, \omega)-\xi_{2}(t, \omega)\right\|^{s} \\
& \quad \leq\left|t-t_{0}\right|\left(C_{1}+C_{2} \sup _{\left|u-t_{0}\right| \leq\left|t-t_{0}\right|} M\left\|\xi_{1}(u, \omega)-\xi_{2}(u, \omega)\right\|^{s}\right),
\end{aligned}
$$

since $d\left(\hat{P}_{*}^{s}\right) \leq 1$.

REMARK 3.7. Let $X_{t}=X_{0}+\hat{P}_{t} a+\hat{P}_{w} v$ and $Y_{t}=Y_{0}+\hat{P}_{t} q+\hat{P}_{w} s$ be two stochastic processes corresponding to $E=I$ and a Banach algebra $H$ over $\mathbf{K}$ in [8, Section 4.3]. Then

$$
X_{u} Y_{u}-X_{t} Y_{t}=\left(X_{u}-X_{t}\right)\left(Y_{u}-Y_{t}\right)+X_{t}\left(Y_{u}-Y_{t}\right)+\left(X_{u}-X_{t}\right) Y_{t},
$$

where $u, t \in T$. Hence $d\left(X_{t} Y_{t}\right)=X_{t} d Y_{t}+\left(d X_{t}\right) Y_{t}+\left(d X_{t}\right)\left(d Y_{t}\right)$. Therefore, $\hat{P}_{X_{t}} Y_{t}=X_{t} Y_{t}-X_{0} Y_{0}-\hat{P}_{Y_{t}} X_{t}-\hat{P}_{\left(X_{t}, Y_{t}\right)}$, which is the non-Archimedean ana$\log$ of the integration by parts formula, where in all terms $X_{t}$ is displayed on the left of $Y_{t}$. For two $C^{1}$ functions $f$ and $g$, we have $(f g)^{\prime}=f^{\prime} g+f g^{\prime}$ or $d(f g)=g d f+f d g$, that is, terms with $(d t)(d t)$ are absent, consequently, $(d t)(d t)=0$. In a particular case $X_{t}=Y_{t}=w_{t}$, this leads to $w_{t}^{2}-w_{0}^{2}-2 \hat{P}_{w_{t}} w_{t}=$ $\hat{P}_{\left(w_{t}, w_{t}\right)}$, where the last term corresponds to $\left(d w_{t}\right)\left(d w_{t}\right) \neq 0$. This means that $d\left(w^{2}\right)=2 w d w+(d w)(d w)$. For $X_{t}=w_{t}$ and $Y_{t}=t$, the integration by parts formula gives $\hat{P}_{w_{t}} t=w_{t} t-\hat{P}_{t} w_{t}-\hat{P}_{\left(t, w_{t}\right)} 1$ such that $\hat{P}_{\left(t, w_{t}\right)} 1=\sum_{j} t_{j}\left[w_{t_{j+1}}-\right.$ $\left.w_{t_{j}}\right]-w_{t} t+\sum_{j} w_{t_{j}}\left[t_{j+1}-t_{j}\right] \neq 0$, for example, for $t=1, w \in C_{0}^{0}(T, H), T=\mathbf{Z}_{p}$ and $t_{0}=0$ this gives $\hat{P}_{\left(t, w_{t}\right)} 1=w_{1}-w_{0}=w_{1}$. Therefore, $(d t)\left(d w_{t}\right) \neq 0$, that is the important difference of the non-Archimedean and classical cases (cf. [10, Exercise 4.3 and Theorem 4.5]).

If $H$ is a Banach space over the local field $\mathbf{K}$ and $f(x, y)=x^{*} y$ is a $\mathbf{K}$-bilinear functional on it, where $x^{*}$ is an image of $x \in H$ under an embedding $H \hookrightarrow H^{*}$ associated with the standard orthonormal base $\left\{e_{j}\right\}$ in $H$, then

$$
\hat{P}_{X_{t}^{*}} Y_{t}=X_{t}^{*} Y_{t}-X_{0}^{*} Y_{0}-\hat{P}_{Y_{t}^{*}} X_{t}-\hat{P}_{\left(X_{t}^{*}, Y_{t}\right)} 1
$$

hence $d\left(X_{t}^{*} Y_{t}\right)=X_{t}^{*} d Y_{t}+\left(d X_{t}^{*}\right) Y_{t}+\left(d X_{t}^{*}\right)\left(d Y_{t}\right)$ and $d\left(w^{*} w\right)=w^{*} d w+$ $\left(d w^{*}\right) w+\left(d w^{*}\right)(d w)$. 
DEFINITION 3.8. If $\xi(t, \omega) \in L^{q}\left(\Omega, \mathscr{F}, \lambda ; C^{0}\left(B_{R}, H\right)\right)=: Z$ is a stochastic process and $T(t, s)$ is a family of bounded linear operators satisfying the following conditions:

(i) $T(t, s): H_{s} \rightarrow H_{t}$, where $H_{s}:=L^{q}\left(\Omega, \mathscr{F}, \lambda ; C^{0}(B(\mathbf{K}, 0,|s|), H)\right)$,

(ii) $T(t, t)=I$,

(iii) $T(t, s) T(s, v)=T(t, v)$ for each $t, s, v \in B_{R}$,

(iv) $M_{s}\left\{\|T(t, s) \eta\|_{H}^{q}\right\} \leq C\|\eta\|_{H}^{q}$ for each $\eta \in H_{s}$, where $C$ is a positive nonrandom constant, $1 \leq q \leq \infty$,

then $T(t, s)$ is called a multiplicative operator functional of the stochastic process $\xi$.

If $T(t, s ; \omega)$ is a system of random variables on $\Omega$ with values in $L(H)$, satisfying a.s. conditions (i), (ii), and (iii) and uniformly by $t, s \in B_{R}$ condition (iv) such that $(T(t, s) \eta)(\omega)=T(t, s ; \omega) \eta(\omega)$, then such multiplicative operator functional is called homogeneous. An operator $A(t)=\lim _{s \rightarrow 0}[T(t, t+s)-I] / s$ is called the generating operator of the evolution family $T(t, v)$. If $T(t, v)=$ $T(t, v ; \omega)$ depends on $\omega$, then $A(t)=A(t ; \omega)$ is also considered as the random variable on $\Omega$ (depending on the parameter $\omega$ ) with values in $L(H)$.

REMARK 3.9. Let $A(t)$ be a linear continuous operator on a Banach space $Y$ over $\mathbf{K}$ such that it strongly and continuously depends on $t \in B(\mathbf{K}, 0, R)$, that is, $A(t) y$ is continuous by $t$ for each chosen $y \in Y$ and $A(t) \in L(Y)$. Then the solution of the differential equation $d x(t) / d t=A(t) x(t), x(s)=x_{0}$, has a solution $x(t)=U(t, s) x(s)$, where $U(t, s)$ is a generating operator such that

$$
U(t, s)=I+\left.\hat{P}_{u} A(u) U(u, s)\right|_{u=s} ^{u=t},
$$

though $x(t)$ may be nonunique, where $x(s)=x_{0}$ is an initial condition, $x, t \in$ $B(\mathbf{K}, 0, R)$. The solution of (3.30) exists by using the method of iterations (see Theorem 3.4).

Indeed, in view of [8, Lemma 2.2], $U(s, s)=I$ and

$$
\frac{d x(t)}{d t}=\frac{\partial U(t, s) x(s)}{\partial t}=A(t) U(t, s) x(s)=A(t) x(t) .
$$

If to consider a solution of the antiderivational equation

$$
V(t, s)=I+\left.\hat{P}_{u} V(t, u) A(u)\right|_{u=s} ^{u=t},
$$

then it is a solution of the Cauchy problem $\partial V(t, s) / \partial s=-V(t, s) A(s), V(t, t)=$ $I$. Therefore, $\partial[V(t, s) U(s, v)] / \partial s=-V(t, s) A(s) U(s, v)+V(t, s) A(s) U(s, v)=$ 0 . Hence $V(t, s) U(s, v)$ is not dependent on $s$; consequently, there exist $U$ and $V$ such that $V(t, s)=U(t, s)$ for each $t, s \in B(\mathbf{K}, 0, R)$. From this it follows that

$$
U(t, s) U(s, u)=U(t, u) \quad \text { for each } s, u, t \in B(\mathbf{K}, 0, R) .
$$

In particular, if $A(t)=A$ is a constant operator, then there exists a solution $U(t, s)=\operatorname{EXP}((t-s) A)$ (see about EXP in [11, Proposition 45.6]). Equation (3.30) 
has a solution under milder conditions, for example, $A(t)$ is weakly continuous, that is, $e^{*} A(t) \eta$ is continuous for each $e^{*} \in Y^{*}$ and $\eta \in Y$. Then $e^{*} U(t, s) \eta$ is differentiable by $t$ and $U(t, s)$ satisfies (3.31) in the weak sense and there exists a weak solution of (3.32) coinciding with $U(t, s)$. If to substitute $A(t)$ on another operator $\tilde{A}(t)$, then for the corresponding evolution operator $\tilde{U}(t, s)$, there is the following inequality:

$$
\|\tilde{U}(t, s)-U(t, s)\| \leq M \tilde{M} \sup _{u \in B(\mathbf{K}, 0, R)}\|\tilde{A}(u)-A(u)\| R
$$

where $M:=1+\sup _{s, t \in B(\mathbf{K}, 0, R)}\|U(t, s)\|$ and $\tilde{M}$ is for $\tilde{U}$.

Proposition 3.10. Let $B(t)$ and two sequences $A_{n}(t)$ and $B_{n}(t)$ be given and strongly continuous on $B(\mathbf{K}, 0, R)$ bounded linear operators, and let $\tilde{U}(t, s)$ be evolution operators corresponding to $\tilde{A}_{n}(t)=A_{n}(t)+B_{n}(t)$, where

$$
\sup _{n \in \mathbb{N}, u \in B(\mathbf{K}, 0, R)}\left\|B_{n}(u)\right\| \leq \sup _{u \in B(\mathbf{K}, 0, R)}\|B(u)\|=C<\infty .
$$

If $M C R<1$, then there exists a sequence $\tilde{U}_{n}(t, s)$ which is also uniformly bounded. If there exists $U_{n}(t, s)$ strongly and uniformly converging to $U(t, s)$ in $B(\mathbf{K}, 0, R)$, then $\tilde{U}_{n}(t, s)$ also can be chosen to be strongly and uniformly convergent.

Proof. From the use of (3.30) and (3.33) iteratively for $U_{n}\left(\sigma_{j+1}(t), \sigma_{j}(t)\right)$, for $U_{n}\left(\sigma_{j}(t), s\right)$, and also for $\tilde{U}_{n}$ and taking $\tilde{U}_{n}-U_{n}$, it follows that

$$
\tilde{U}_{n}(t, s)=U_{n}(t, s)+\left.\hat{P}_{v} U_{n}(t, v) B_{n}(v) \tilde{U}_{n}(v, s)\right|_{v=s} ^{v=t} \quad \text { for each } n \in \mathbb{N} .
$$

Therefore, $\left\|\tilde{U}_{n}(t, s)\right\| \leq M+M C \sup _{v}\left\|\tilde{U}_{n}(v, s)\right\| R$, hence $\left\|\tilde{U}_{n}(t, s)\right\| \leq M /[1-$ $M C R]$ since $M C R<1$. If $\lim _{n} x_{n}=x$ in $Y$ and $U_{n}(t, s) x$ is uniformly convergent to $U(t, s) x$, then for each $\epsilon>0$ there exist $\delta>0$ and $m \in \mathbb{N}$ such that $\sup _{t, s \in B(\mathrm{~K}, 0, R)}\left\|U_{n}(t+h, s+v) x_{n}-U_{n}(t, s) x_{n}\right\|<\epsilon$, for each $n>m$, and $\max (|h|,|v|)<\delta$ due to equality (3.36).

Proposition 3.11. Let $a, a_{m-l+b, l}$, and $E$ be the same as in Theorem 3.4. Then Theorem 3.4(i) has the unique solution $\xi$ in $B_{R}$ for each initial value $\xi\left(t_{0}, \omega\right) \in L^{q}(\Omega, \mathscr{F}, \lambda ; H)$ and it can be represented in the following form:

$$
\xi(t, \omega)=T\left(t, t_{0} ; \omega\right) \xi\left(t_{0} ; \omega\right),
$$

where $T(t, v ; \omega)$ is the multiplicative operator functional.

Proof. In view of Theorem 3.4, Definition 3.8, Remark 3.9, and Proposition 3.10 with the use of a parameter $\omega \in \Omega$, the statement of Proposition 3.11 follows.

3.3. Now we consider the case $J\left(C_{0}^{0}(T, H)\right) \subset C^{1}(T, H)$ (see Proposition 3.5), for example, the standard Wiener process. 
COROLLARY 3.12. Let a function $f(t, x)$ satisfy conditions of $[8$, Theorem 4.7], then a generating operator of an evolution family $T(t, v)$ of a stochastic process $\eta=f(t, \xi(t, \omega))$ is given by the following equation:

$$
\begin{aligned}
& A(t) \eta(t)=f_{t}^{\prime}(t, \xi(t, \omega)) \\
& +f_{x}^{\prime}(t, \xi(t, \omega)) \circ a(t, \omega) \\
& +f_{x}^{\prime}(t, \xi(t, \omega)) \circ E(t, \omega) w^{\prime}{ }_{t}(t, \omega) \\
& +\sum_{m+b \geq 2,0 \leq m \in \mathbf{Z}, 0 \leq b \in \mathbf{Z}}((m+b) !)^{-1} \sum_{l=0}^{m}\left(\begin{array}{c}
m+b \\
m
\end{array}\right)\left(\begin{array}{c}
m \\
l
\end{array}\right) \\
& \times\left\{( b + m - l ) \left(\hat { P } _ { u ^ { b + m - l - 1 } , w ( u , w ) ^ { l } } \left[\left(\frac{\partial^{(m+b)} f}{\partial u^{b} \partial x^{m}}\right)\right.\right.\right. \\
& \left.\left.\times(u, \xi(u, \omega)) \circ\left(I^{\otimes b} \otimes a^{\otimes(m-l)} \otimes E^{\otimes l}\right)\right]\right)\left.\right|_{u=t} \\
& +l\left(\hat { P } _ { u ^ { b + m - l } , w ( u , \omega ) ^ { l - 1 } } \left[\left(\frac{\partial^{(m+b)} f}{\partial u^{b} \partial x^{m}}\right)(u, \xi(u, \omega))\right.\right. \\
& \left.\left.\left.\circ\left(I^{\otimes b} \otimes a^{\otimes(m-l)} \otimes E^{\otimes(l-1)}\right)\right] E w_{u}^{\prime}(u, \omega)\right)\left.\right|_{u=t}\right\} .
\end{aligned}
$$

Proof. In view of [8, Theorem 4.7] and Proposition 3.11, there exists a generating operator of an evolution family. From [8, Lemma 2.2 and formula (4.14)], the statement of this corollary follows.

REMARK 3.13. If $f(t, x)$ satisfies conditions either of [8, Section 4.3] or of [8, Corollary 4.6], then formula (3.38) takes simpler forms since the corresponding terms vanish.

ACKNOWLEDGment. The author is sincerely grateful to I. V. Volovich for his interest in this work and fruitful discussions.

\section{REFERENCES}

[1] Y. Amice, Interpolation p-adique, Bull. Soc. Math. France 92 (1964), 117-180.

[2] Yu. L. Dalecky and S. V. Fomin, Measures and Differential Equations in InfiniteDimensional Space, Mathematics and Its Applications (Soviet Series), vol. 76, Kluwer Academic Publishers, Dordrecht, 1991.

[3] J. M. G. Fell and R. S. Doran, Representations of *-Algebras, Locally Compact Groups, and Banach *-Algebraic Bundles. Vol. 1, Pure and Applied Mathematics, vol. 125, Academic Press, Massachusetts, 1988.

[4] _ Representations of *-Algebras, Locally Compact Groups, and Banach * Algebraic Bundles. Vol. 2, Pure and Applied Mathematics, vol. 126, Academic Press, Massachusetts, 1988.

[5] S. V. Ludkovsky, The non-Archimedean analogs of the BochnerKolmogorov, Minlos-Sazonov and Kakutani theorems, preprint, 2000, http://arxiv.org/abs/math.FA/0010230. 
[6] _ Quasi-invariant and pseudo-differentiable measures on a non-Archimedean Banach space. I. Real-valued measures, preprint, 1996, http://arxiv.org/abs/math.GM/0106169.

[7] _ Quasi-invariant and pseudo-differentiable measures on a nonArchimedean Banach space. II. Measures with values in non-Archimedean fields, preprint, 1996, http://arxiv.org/abs/math.GM/0106170.

[8] _ Stochastic processes on non-Archimedean Banach spaces, Int. J. Math. Math. Sci. 2003 (2003), no. 21, 1341-1363.

[9] S. V. Ludkovsky and B. Diarra, Spectral integration and spectral theory for nonArchimedean Banach spaces, Int. J. Math. Math. Sci. 31 (2002), no. 7, 421442.

[10] B. Øksendal, Stochastic Differential Equations, Universitext, Springer-Verlag, Berlin, 1995.

[11] W. H. Schikhof, Ultrametric Calculus, Cambridge Studies in Advanced Mathematics, vol. 4, Cambridge University Press, Cambridge, 1984.

[12] A. C. M. van Rooij, Non-Archimedean Functional Analysis, Monographs and Textbooks in Pure and Applied Math., vol. 51, Marcel Dekker, New York, 1978.

[13] V. S. Vladimirov, I. V. Volovich, and E. I. Zelenov, $p$-Adic Analysis and Mathematical Physics, Nauka, Moscow, 1994 (Russian).

S. V. Ludkovsky: Theoretical Department, Institute of General Physics, 38 Vavilov Street, Moscow 119991, GSP-1, Russia

E-mail address: 1udkovsk@fp 1.gpi .ru 


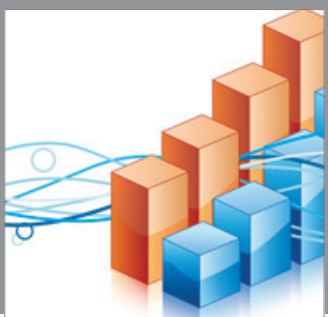

Advances in

Operations Research

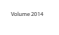

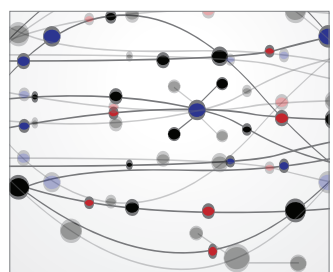

\section{The Scientific} World Journal
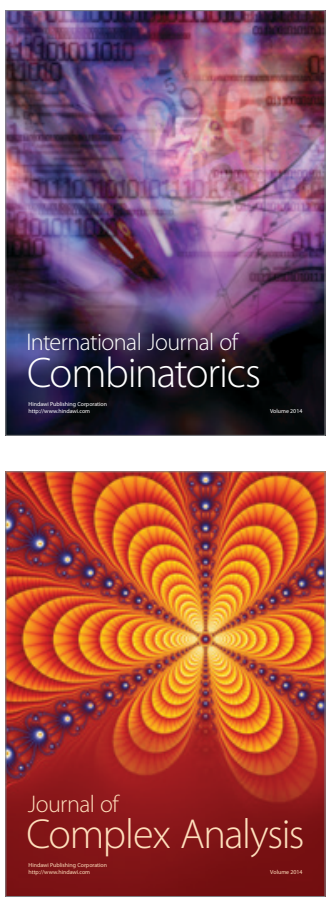

International Journal of

Mathematics and

Mathematical

Sciences
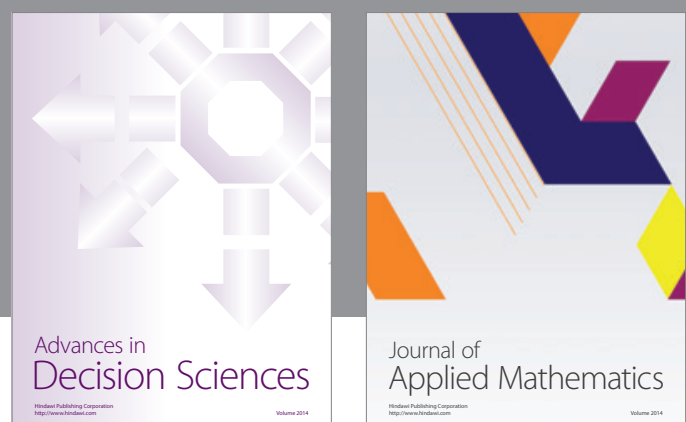

Journal of

Applied Mathematics
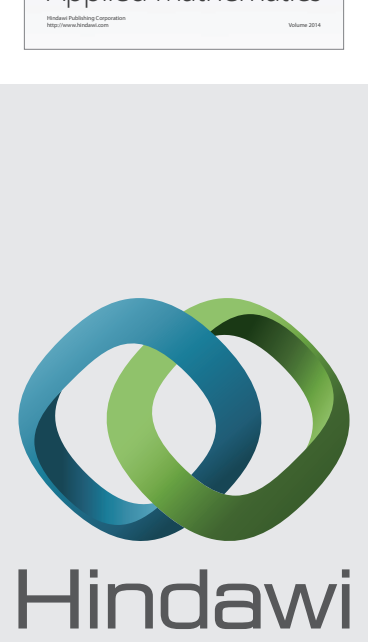

Submit your manuscripts at http://www.hindawi.com
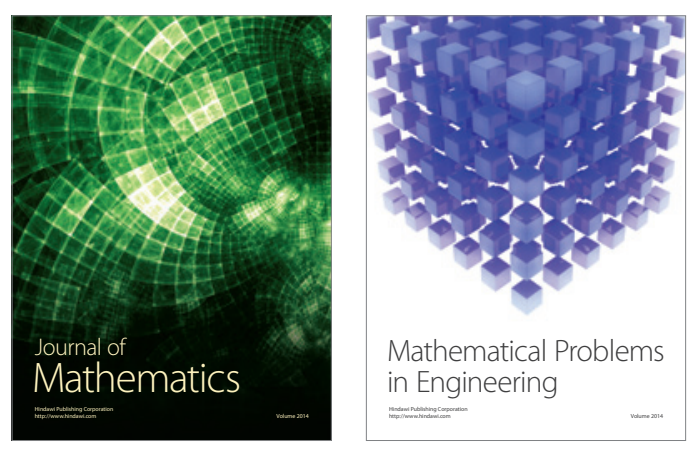

Mathematical Problems in Engineering
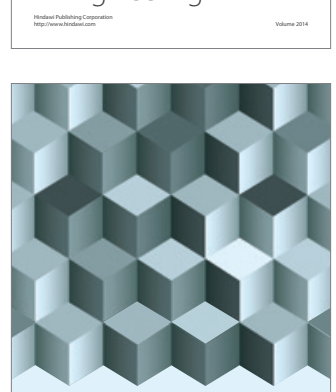

Journal of

Function Spaces
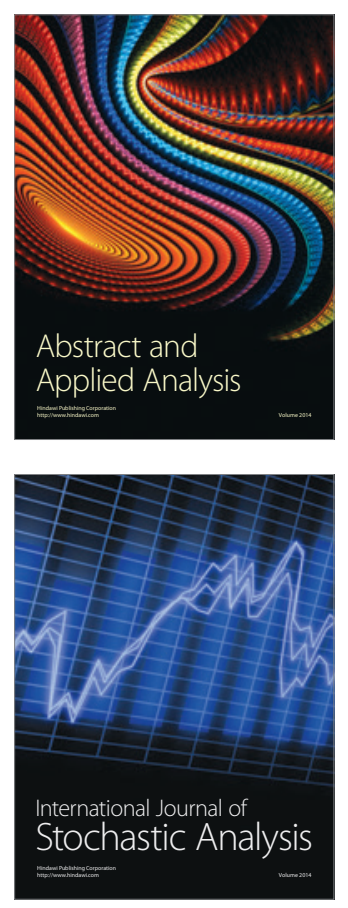

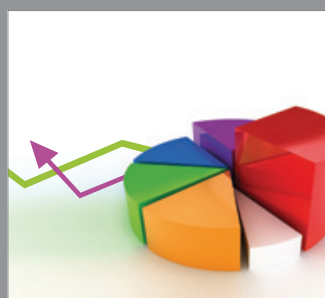

ournal of

Probability and Statistics

Promensencen
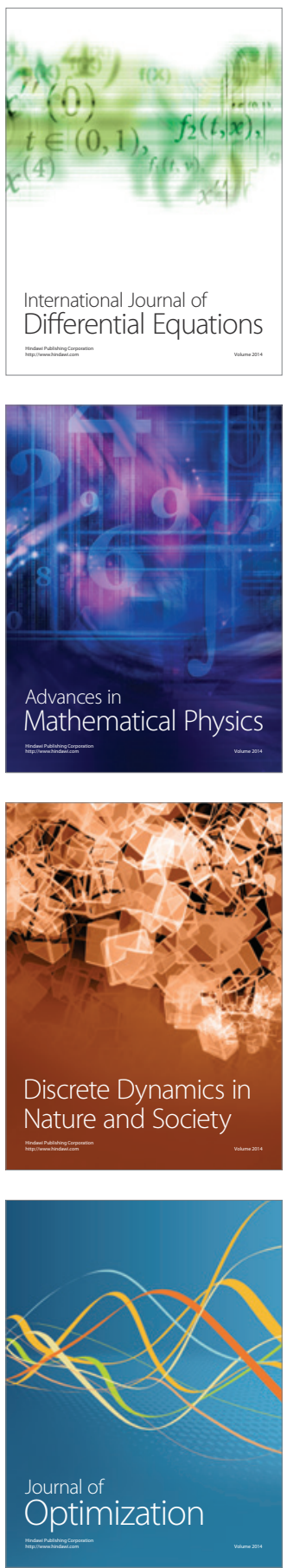